\title{
Inpatient costs and blood transfusion rates of sarcopenic patients following thoracolumbar spine surgery
}

\author{
Steven L. Bokshan, MD, ${ }^{2,3}$ Alex Han, BS, ${ }^{3}$ J. Mason DePasse, MD, ${ }^{2,3}$ Stephen E. Marcaccio, MD, ${ }^{3}$ \\ Adam E. M. Eltorai, MSc, ${ }^{3}$ and Alan H. Daniels, MD ${ }^{1-3}$ \\ 'Division of Spine Surgery, ${ }^{2}$ Department of Orthopaedic Surgery, and ${ }^{3}$ Warren Alpert Medical School of Brown University, \\ Providence, Rhode Island
}

\begin{abstract}
OBJECTIVE Sarcopenia, the muscle atrophy associated with aging and disease progression, accounts for nearly $\$ 18.5$ billion in health care expenditures annually. Given the high prevalence of sarcopenia in patients undergoing orthopedic surgery, the goal of this study was to assess the impact of sarcopenia on inpatient costs following thoracolumbar spine surgery.
\end{abstract}

METHODS Patients older than 55 years undergoing thoracolumbar spine surgery from 2003 to 2015 were retrospectively analyzed. Sarcopenia was measured using total psoas area at the L-4 vertebra on perioperative CT scans. Hospital billing data were used to compare inpatient costs, transfusion rate, and rate of advanced imaging utilization.

RESULTS Of the 50 patients assessed, 16 were sarcopenic. Mean total hospital costs were 1.75-fold greater for sarcopenic patients compared with nonsarcopenic patients $(\$ 53,128$ vs $\$ 30,292, p=0.04)$. Sarcopenic patients were 2.1 times as likely to require a blood transfusion $(43.8 \%$ vs $20.6 \%, p=0.04)$. Sarcopenic patients had a 2.6 -fold greater usage of advanced imaging $(68.8 \%$ vs $26.5 \%, p=0.002)$ with associated higher diagnostic imaging costs $(\$ 2452$ vs $\$ 801, p=$ 0.01). Sarcopenic patients also had greater pharmacy, laboratory, respiratory care, and emergency department costs.

CONCLUSIONS This study is the first to show that sarcopenia is associated with higher postoperative costs and rates of blood transfusion following thoracolumbar spine surgery. Measuring the psoas area may represent a strategy for predicting perioperative costs in spine surgery patients.

https://thejns.org/doi/abs/10.3171/2017.5.SPINE17171

KEY WORDS sarcopenia; frailty; thoracolumbar spine surgery; inpatient costs; lumbar; thoracic

$\mathrm{S}$ ARCOPENIA is the muscle atrophy associated with aging and disease progression. ${ }^{6}$ This condition is highly prevalent in the elderly population, with older adults losing up to $15 \%$ of their total muscle mass during their 7 th decade of life. ${ }^{19}$ In addition to being an independent predictor of fall risk, hospital-acquired infection, and all-cause mortality, ${ }^{1,5,16}$ sarcopenia has been shown to significantly increase inpatient costs following elective general surgery. ${ }^{14}$

Over the last 2 decades, Medicare spending on lumbar spine surgery has risen nearly $500 \%$, reaching a cost of
$\$ 482$ million annually. ${ }^{28}$ As these costs have continued to grow, so too has the need for cost-effective surgical practices and appropriate patient selection. ${ }^{26}$ The assessment of sarcopenia prior to surgery may represent one such risk stratification tool. To put the impact of sarcopenia in perspective, it has been estimated that a $10 \%$ decrease in the prevalence of sarcopenia nationally could amount to health care cost savings of $\$ 1.1$ billion annually in the US. ${ }^{11}$

While sarcopenia has been identified as an independent predictor of higher postoperative costs and poorer outcomes following general surgery, ${ }^{7,8,14,24,27}$ few studies have 
measured the impact of sarcopenia on inpatient surgical costs following orthopedic surgery, despite the fact that as many as $44 \%$ of orthopedic patients may meet the criteria for sarcopenia. ${ }^{13}$

In this investigation, we examined the effect of sarcopenia on inpatient surgical costs following thoracolumbar spine surgery. In addition to the total inpatient cost, a subanalysis was performed to examine which aspects of the hospitalization were costlier for sarcopenic patients. We hypothesized that sarcopenia would be associated with a greater overall cost of hospitalization following thoracolumbar spine surgery.

\section{Methods \\ Study Design}

Institutional review board approval from Rhode Island Hospital was obtained prior to initiating this investigation. All patients undergoing thoracolumbar decompression or decompression with fusion at a single institution from 2003 to 2015 were analyzed. All surgical indications were considered for inclusion. Patients were included if they were older than 55 years, if there was a perioperative CT scan available for measuring psoas muscle cross-sectional area (performed an average of 4.3 months prior to date of surgery; range 0-21.4 months), and if inpatient cost data were available from the hospital's billing department. Patients were excluded if a CT scan had been performed more than 2 years prior to a patient's surgery date, or if the patient's inpatient cost data were unavailable.

\section{Measurement of Sarcopenia}

All sarcopenia measurements were performed using axial views of the psoas muscle at the L-4 vertebra on $\mathrm{CT}$. The cross-sectional areas of the left and right psoas muscles at the level of the transverse process of L-4 were measured manually using OsiriX imaging software (Pixmeo), with fat stranding observed on CT used to exclude fat tissue from psoas muscle measurement. Measurements were made using the axial image in which both transverse processes were fully visualized to improve objectivity and reproducibility of the measurement, as previously described. ${ }^{21}$ The cross-sectional areas were then added to calculate the L-4 total psoas area (TPA). ${ }^{9}$ A single investigator blinded to patient diagnosis and outcomes calculated the TPA two separate times for each patient, in a random order on two different occasions, with the two values subsequently averaged. Patients were diagnosed with sarcopenia if they fell into the lowest tertile for their sex-specific TPA.

\section{Outcome Variables}

The primary outcome variable was the total inpatient hospitalization cost, which included the cost of the surgical procedure and all costs incurred during the inpatient postoperative period. Secondary outcomes measures included the following individual costs: surgical cost (cost of operating room time, instrumentation, and perioperative nursing care), cost of occupying a hospital bed (nursing care for a routine bed, step-down bed, or intensive care unit [ICU] bed), blood bank, laboratory studies, diagnos- tic imaging, pharmacy costs, respiratory care, endoscopy, emergency department cost, rehabilitation services (both physical and occupational therapists), intravenous therapy (cost of fluid resuscitation, venous access), and central supply (cost of all medical-grade equipment not routinely available on the hospital floor). Costs were obtained from the hospital charge center prior to being negotiated by insurance, with cost data adjusted for year-to-year inflation by the cost center. All costs were based on a standardized hospital registrar, completely independent of insurance type or reimbursement rates. Additional secondary outcomes included requirements for blood transfusions and advanced imaging (defined as CT, MRI, or ultrasonography).

Preoperative health and comorbidity burden was measured using the Charlson Comorbidity Index (CCI), a well-validated index used to account for complex medical comorbidities such as end-organ disease (e.g., end-stage renal disease) and presence of malignancy (localized and metastatic). ${ }^{25}$ Patient charts were reviewed in detail to identify medical comorbidities based on ICD-10 codes, which were subsequently used for calculation of the CCI as previously described. ${ }^{4,22}$

\section{Statistical Analysis}

A Student t-test was used to compare mean differences in total hospital costs and for each individual department cost between sarcopenic and nonsarcopenic patients. A zscore was used to assess for differences in the proportion of patients receiving blood transfusion or advanced imaging for sarcopenic versus nonsarcopenic patients. A cutoff of $p=0.05$ was used to determine significance for all tests (SPSS version 21, IBM Corp.).

\section{Results}

Fifty patients met inclusion criteria for the study, with 16 (8 men and 8 women) falling into the lowest tertile for sex-specific L-4 TPA and thus diagnosed with sarcopenia. Follow-up duration ranged from 6 days (died in hospital) to 12.7 years, with an average of 4.6 years. Indications for surgery were spinal stenosis in 35 patients (4 secondary to metastasis), degenerative scoliosis in 4 patients, epidural abscess or discitis in 5, acute fracture requiring operative fixation in 4 (1 secondary to metastasis), and radiculopathy in 2 (Table 1). All 5 patients requiring an operation for spinal metastasis were sarcopenic. However, there was no significant difference between the proportion of sarcopenic and nonsarcopenic patients with any diagnosed malignancy ( 8 vs 9 patients, respectively; $\mathrm{p}=0.1$ ). Sarcopenic patients were significantly older than nonsarcopenic patients with a mean age of 76.6 years compared with 70.8 years, respectively $(\mathrm{p}=0.027)$. There was no statistically significant difference in mean CCI (3.6 vs 3.2, $\mathrm{p}=0.59$ ) for sarcopenic patients compared with nonsarcopenic patients.

The average total hospital cost was 1.75 -fold greater for sarcopenic versus nonsarcopenic patients $(\$ 53,128$ vs $\$ 30,292, p=0.04$; Table 2). There was no significant difference in total surgical costs (inclusive of operating room time, instrumentation cost, and cost of perioperative care) for sarcopenic versus nonsarcopenic patients $(\$ 23,515$ vs 
TABLE 1. Baseline patient characteristics by sarcopenic status

\begin{tabular}{|c|c|c|c|}
\hline Variable & Sarcopenic & Nonsarcopenic & $p$ Value \\
\hline \multicolumn{4}{|l|}{ Sex } \\
\hline Male & 8 & 18 & \\
\hline Female & 8 & 16 & \\
\hline Mean age \pm SD (yrs) & $76.6 \pm 2.2$ & $70.8 \pm 1.4$ & 0.027 \\
\hline Mean L-4 TPA \pm SD $\left(\mathrm{mm}^{2}\right)$ & $1297.6 \pm 105.2$ & $2283.4 \pm 123.5$ & $<0.001$ \\
\hline Mean $\mathrm{CCl} \pm \mathrm{SD}$ & $3.6 \pm 0.7$ & $3.2 \pm 0.4$ & 0.59 \\
\hline \multicolumn{4}{|l|}{ Preop diagnosis } \\
\hline $\begin{array}{l}\text { Spinal stenosis (metas- } \\
\text { tasis) }\end{array}$ & $11(4)$ & 24 & \\
\hline Degenerative scoliosis & 0 & 4 & \\
\hline Epidural abscess/discitis & 2 & 3 & \\
\hline $\begin{array}{l}\text { Acute fracture (metas- } \\
\text { tasis) }\end{array}$ & $3(1)$ & 1 & \\
\hline Radiculopathy & 0 & 2 & \\
\hline
\end{tabular}

All data given as number of patients unless otherwise indicated.

$\$ 19,140, p=0.31)$. While the cost of occupying a routine hospital bed was greater for sarcopenic patients $(\$ 10,522$ vs $\$ 4934, p=0.002$ ), there was no significant difference in cost for step down-level beds ( $\$ 2831$ vs $\$ 714, \mathrm{p}=0.11$ ) or ICU-level beds ( $\$ 6520$ vs $\$ 2442, p=0.12$ ) for sarcopenic patients compared with nonsarcopenic patients.

Blood bank cost was significantly higher for sarcopenic patients compared with nonsarcopenic patients $(\$ 960$ vs $\$ 167, p=0.01)$. Sarcopenic patients were 2.1 times as likely to require blood transfusion $(43.8 \%$ vs $20.6 \%$, p = 0.04 ), with a significantly greater number of units transfused than nonsarcopenic patients (1.4 vs 0.3 units, $\mathrm{p}=$ $0.02)$. Similarly, diagnostic imaging costs were significantly greater for sarcopenic patients ( $\$ 2452$ vs $\$ 801, \mathrm{p}=0.01$ ), with a 2.6 -fold greater usage of advanced imaging $(68.8 \%$ vs $26.5 \%, p=0.002$ ).

With regard to the remaining aspects of hospitalization, sarcopenia was associated with a higher cost for pharmacy $(\$ 2128$ vs $\$ 609, \mathrm{p}<0.001)$, laboratory studies $(\$ 1128$ vs $\$ 274, \mathrm{p}=0.004)$, respiratory care $(\$ 649$ vs $\$ 225, \mathrm{p}=$ $0.04)$, and the emergency department ( $\$ 375$ vs $\$ 147, \mathrm{p}=$ $0.04)$. However, there were nonsignificant differences in costs for rehabilitation services $(\$ 123$ vs $\$ 55, \mathrm{p}=0.08)$, intravenous therapy ( $\$ 249$ vs $\$ 104, p=0.14)$, central supply ( $\$ 111$ vs $\$ 6, p=0.18$ ), and endoscopy ( $\$ 123$ vs $\$ 55, p$ $=0.31$ ) between sarcopenic and nonsarcopenic patients.

\section{Discussion}

Sarcopenia is a common condition in older age and may account for an additional $\$ 18.5$ billion in health care expenditures annually. ${ }^{25}$ Our previous study of sarcopenia in patients who underwent thoracolumbar spine surgery demonstrated that sarcopenic patients had a significantly increased risk of death, in-hospital complications, and discharge to rehabilitation facilities, as well as longer length of hospital stay. ${ }^{2}$ While previous studies have shown that sarcopenia increases the cost of elective general surgery by nearly $\$ 10,000$ per $\mathrm{cm}^{2}$ of psoas area, this study is the
TABLE 2. Cost comparison for sarcopenic patients compared to nonsarcopenic patients

\begin{tabular}{lccc}
\hline $\begin{array}{c}\text { Cost } \\
\text { Variable }\end{array}$ & $\begin{array}{c}\text { Sarcopenic } \\
(\$)^{*}\end{array}$ & $\begin{array}{c}\text { Nonsarcopenic } \\
(\$)^{*}\end{array}$ & $\begin{array}{c}p \\
\text { Value }\end{array}$ \\
\hline Total inpatient cost & $53,128 \pm 10,612$ & $30,292 \pm 6535$ & $0.04 \dagger$ \\
\hline Routine hospital bed & $10,522 \pm 1618$ & $4934 \pm 760$ & $0.002 \dagger$ \\
\hline Blood bank & $960 \pm 311$ & $167 \pm 54$ & $0.01 \dagger$ \\
\hline Diagnostic imaging $\neq$ & $2452 \pm 529$ & $801 \pm 262$ & $0.01 \dagger$ \\
\hline Pharmacy & $2128 \pm 356$ & $609 \pm 93$ & $<0.001 \dagger$ \\
\hline Laboratory studies & $1128 \pm 281$ & $274 \pm 78$ & $0.004 \dagger$ \\
\hline Respiratory care & $649 \pm 205$ & $225 \pm 110$ & $0.04 \dagger$ \\
\hline Emergency department & $375 \pm 114$ & $147 \pm 57$ & $0.04 \dagger$ \\
\hline Surgery & $23,515 \pm 7193$ & $19,140 \pm 4901$ & 0.31 \\
\hline ICU bed & $6520 \pm 3129$ & $2442 \pm 1314$ & 0.12 \\
\hline Step-down bed & $2831 \pm 1648$ & $714 \pm 404$ & 0.11 \\
\hline
\end{tabular}

* Data given as mean \pm SEM.

$\dagger$ Statistically significant cost differences.

‡ MRI, CT, or ultrasonography.

first to demonstrate that sarcopenia is associated with significantly higher costs following thoracolumbar spine surgery. ${ }^{8}$ Specifically, the total hospitalization cost for sarcopenic patients was nearly $\$ 23,000$ greater per patient. This finding is particularly salient given that sarcopenia rates are as high as $44.1 \%$ in certain orthopedic populations. ${ }^{13}$

Interestingly, there was no significant difference in the total operative cost; the biggest differences in costs were in occupying a routine hospital bed, receiving blood, laboratory testing, advanced diagnostic imaging, and respiratory care. Therefore, it appears that the largest cost drivers during hospitalization are the need for closer monitoring and the interventions for blood loss and other surgical complications. These nonsurgical costs accounted for more than $50 \%$ of the total cost in sarcopenic patients but less than 35\% in nonsarcopenic patients (Fig. 1). These findings are consistent with numerous previous investigations demonstrating that sarcopenia is an independent predictor of postoperative complications following abdominal and emergency surgery.,14,15 Likewise, sarcopenia has also been associated with reduced left ventricular ejection fractions and respiratory failure..$^{12,20}$

It is especially important that sarcopenic patients were 2.1 times more likely to receive a blood transfusion compared with nonsarcopenic patients. Not only has blood transfusion been shown to increase the potential for postoperative infection and the average length of ICU stay following spine surgery, but it also exposes patients to transfusion-related acute lung injury and transfusion-associated circulatory overload, both of which may be fatal to the sarcopenic patient. ${ }^{10}$

This study had several potential limitations. One limitation is that only the L-4 TPA was used to define sarcopenia. Other investigations have used Hounsfield units on CT scan, quadriceps area, dual energy x-ray absorptiometry, or bioelectrical impedance analysis in combination with measures of muscle strength (handgrip) and physical performance (gait speed)., ${ }^{3,17,23}$ L-4 TPA was used in this 


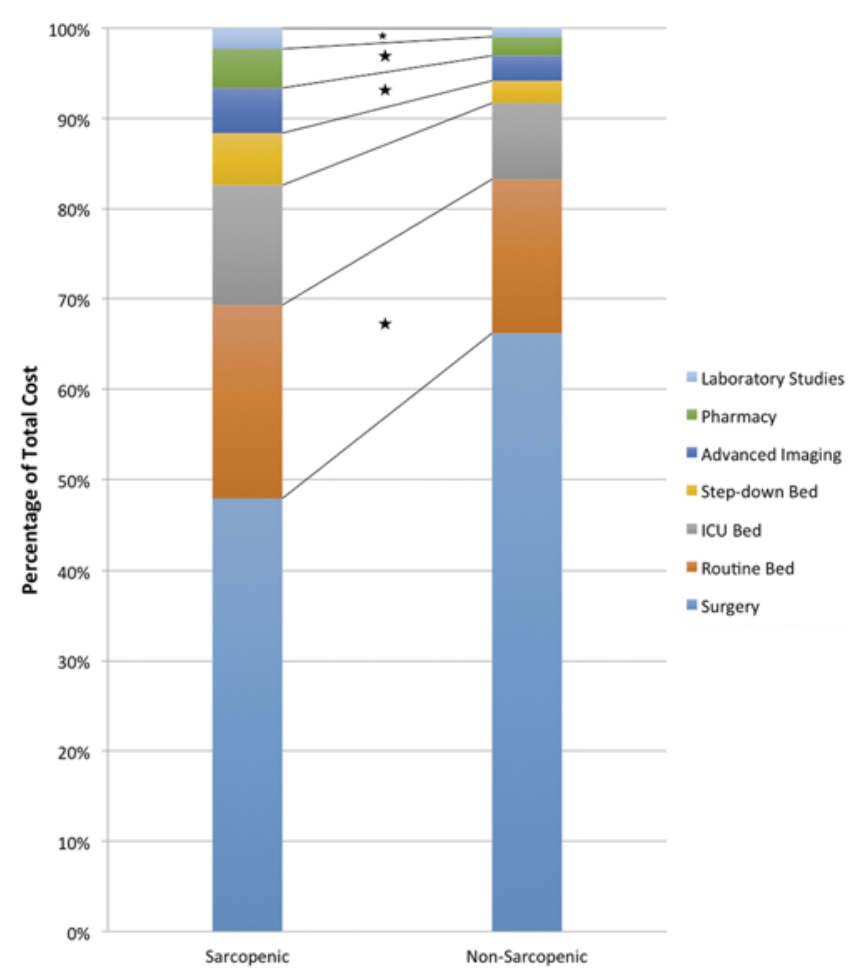

FIG. 1. Percentage of total cost for the 7 largest cost drivers during hospitalization for thoracolumbar spine surgery. A star denotes statistically significant differences. Figure is available in color online only.

study given the wide availability of CT scans in spine surgery patients, as well as the strong objectivity and reproducibility of this measure. ${ }^{9}$ In addition, because CT scans were obtained perioperatively, we could not exclude the possibility that patients may have developed sarcopenia due to the operation itself. Furthermore, due to a lack of data regarding patient body mass index, we were not able to identify patients with sarcopenic obesity, which has been associated with worse outcomes. ${ }^{18}$ Another potential limitation is that sarcopenic patients in our study were significantly older than nonsarcopenic patients, and that all 5 patients with spinal metastasis were sarcopenic. This may have contributed to the higher cost of care associated with sarcopenia, although it should be noted that sarcopenic and nonsarcopenic patients in our study did not significantly differ in mean CCI score, which adjusts for age and accounts for comorbidities including metastasis. Finally, our study retrospectively analyzed a patient cohort from a single institution, and accounted for only direct patient costs. Future studies would be strengthened by analyzing indirect costs in addition to prospectively studying a larger sample size derived from multiple centers.

\section{Conclusions}

This study demonstrates that sarcopenia is associated with higher total postoperative costs following thoracolumbar surgery, in addition to higher costs for both routine laboratory tests and advanced diagnostic imaging. Given the growing importance of cost containment and an in- creasingly outcomes-based surgical climate, measuring sarcopenia represents one potential strategy for screening spine surgery patients and predicting surgical cost, especially considering the widespread use of lumbar CT scans in this setting.

\section{References}

1. Batsis JA, Mackenzie TA, Barre LK, Lopez-Jimenez F, Bartels SJ: Sarcopenia, sarcopenic obesity and mortality in older adults: results from the National Health and Nutrition Examination Survey III. Eur J Clin Nutr 68:1001-1007, 2014

2. Bokshan SL, Han AL, DePasse JM, Eltorai AEM, Marcaccio SE, Palumbo MA, et al: Effect of sarcopenia on postoperative morbidity and mortality after thoracolumbar spine surgery. Orthopedics 39:e1159-e1164, 2016

3. Buchner DM, Larson EB, Wagner EH, Koepsell TD, de Lateur BJ: Evidence for a non-linear relationship between leg strength and gait speed. Age Ageing 25:386-391, 1996

4. Charlson M, Szatrowski TP, Peterson J, Gold J: Validation of a combined comorbidity index. J Clin Epidemiol 47:12451251, 1994

5. Cosquéric G, Sebag A, Ducolombier C, Thomas C, Piette F, Weill-Engerer S: Sarcopenia is predictive of nosocomial infection in care of the elderly. Br J Nutr 96:895-901, 2006

6. Cruz-Jentoft AJ, Baeyens JP, Bauer JM, Boirie Y, Cederholm T, Landi F, et al: Sarcopenia: European consensus on definition and diagnosis. Age Ageing 39:412-423, 2010

7. Du Y, Karvellas CJ, Baracos V, Williams DC, Khadaroo RG: Sarcopenia is a predictor of outcomes in very elderly patients undergoing emergency surgery. Surgery 156:521-527, 2014

8. Englesbe MJ, Lee JS, He K, Fan L, Schaubel DE, Sheetz KH, et al: Analytic morphomics, core muscle size, and surgical outcomes. Ann Surg 256:255-261, 2012

9. Englesbe MJ, Patel SP, He K, Lynch RJ, Schaubel DE, Harbaugh C, et al: Sarcopenia and mortality after liver transplantation. J Am Coll Surg 211:271-278, 2010

10. Hu SS: Blood loss in adult spinal surgery. Eur Spine J 13 (Suppl 1):S3-S5, 2004

11. Janssen I, Shepard DS, Katzmarzyk PT, Roubenoff R: The healthcare costs of sarcopenia in the United States. J Am Geriatr Soc 52:80-85, 2004

12. Jeon YK, Shin MJ, Kim MH, Mok JH, Kim SS, Kim BH, et al: Low pulmonary function is related with a high risk of sarcopenia in community-dwelling older adults: the Korea National Health and Nutrition Examination Survey (KNHANES) 2008-2011. Osteoporos Int 26:2423-2429, 2015

13. Ji HM, Won YY, Kang BM, Park YS, Han MH: Sarcopenia in patients scheduled to undergo orthopaedic surgery. Bone Abstr 3:PP204, 2014 (Abstract)

14. Kirk PS, Friedman JF, Cron DC, Terjimanian MN, Wang SC, Campbell DA, et al: One-year postoperative resource utilization in sarcopenic patients. J Surg Res 199:51-55, 2015

15. Kuroki LM, Mangano M, Allsworth JE, Menias CO, Massad LS, Powell MA, et al: Pre-operative assessment of muscle mass to predict surgical complications and prognosis in patients with endometrial cancer. Ann Surg Oncol 22:972979, 2015

16. Landi F, Liperoti R, Russo A, Giovannini S, Tosato M, Capoluongo E, et al: Sarcopenia as a risk factor for falls in elderly individuals: results from the ilSIRENTE study. Clin Nutr 31:652-658, 2012

17. Lauretani F, Russo CR, Bandinelli S, Bartali B, Cavazzini C, Di Iorio A, et al: Age-associated changes in skeletal muscles and their effect on mobility: an operational diagnosis of sarcopenia. J Appl Physiol (1985) 95:1851-1860, 2003

18. Mei KL, Batsis JA, Mills JB, Holubar SD: Sarcopenia and sarcopenic obesity: do they predict inferior oncologic out- 
comes after gastrointestinal cancer surgery? Perioper Med (Lond) 5:30, 2016

19. Melton LJ III, Khosla S, Crowson CS, O’Connor MK, O'Fallon WM, Riggs BL: Epidemiology of sarcopenia. J Am Geriatr Soc 48:625-630, 2000

20. Narumi T, Watanabe T, Kadowaki S, Takahashi T, Yokoyama M, Kinoshita D, et al: Sarcopenia evaluated by fat-free mass index is an important prognostic factor in patients with chronic heart failure. Eur J Intern Med 26:118-122, 2015

21. Psutka SP, Carrasco A, Schmit GD, Moynagh MR, Boorjian SA, Frank I, et al: Sarcopenia in patients with bladder cancer undergoing radical cystectomy: impact on cancer-specific and all-cause mortality. Cancer 120:2910-2918, 2014

22. Quan H, Sundararajan V, Halfon P, Fong A, Burnand B, Luthi JC, et al: Coding algorithms for defining comorbidities in ICD-9-CM and ICD-10 administrative data. Med Care 43:1130-1139, 2005

23. Rajaee SS, Bae HW, Kanim LEA, Delamarter RB: Spinal fusion in the United States: analysis of trends from 1998 to 2008. Spine (Phila Pa 1976) 37:67-76, 2012

24. Reisinger KW, van Vugt JLA, Tegels JJW, Snijders C, Hulsewé KWE, Hoofwijk AGM, et al: Functional compromise reflected by sarcopenia, frailty, and nutritional depletion predicts adverse postoperative outcome after colorectal cancer surgery. Ann Surg 261:345-352, 2015

25. Sayer AA: Sarcopenia. BMJ 341:c4097, 2010

26. Stammen LA, Stalmeijer RE, Paternotte E, Oudkerk Pool A, Driessen EW, Scheele F, et al: Training physicians to provide high-value, cost-conscious care: a systematic review. JAMA 314:2384-2400, 2015
27. Tan BHL, Birdsell LA, Martin L, Baracos VE, Fearon KCH: Sarcopenia in an overweight or obese patient is an adverse prognostic factor in pancreatic cancer. Clin Cancer Res 15:6973-6979, 2009

28. Weinstein JN, Lurie JD, Olson PR, Bronner KK, Fisher ES: United States' trends and regional variations in lumbar spine surgery: 1992-2003. Spine (Phila Pa 1976) 31:2707-2714, 2006

\section{Disclosures}

Dr. Daniels reports being a paid consultant for DePuy, Globus Medical, Orthofix, SpineArt, and Stryker, and an unpaid consultant for Osseus; receiving research support for the study from Orthofix; and receiving financial or material support from DePuy, Medtronic Sofamor Danek, and Stryker.

\section{Author Contributions}

Conception and design: all authors. Acquisition of data: all authors. Analysis and interpretation of data: all authors. Drafting the article: all authors. Critically revising the article: all authors. Reviewed submitted version of manuscript: Daniels, Bokshan, DePasse, Eltorai. Statistical analysis: Bokshan.

\section{Correspondence}

Alan H. Daniels, Department of Orthopaedic Surgery, Brown University, Warren Alpert Medical School, 100 Butler Dr., Providence, RI 02906. email: alandanielsmd@gmail.com. 